\title{
Axiomatic Holonomy Maps and Generalized YANG-Mills Moduli Space
}

\author{
Piotr M. HAJAC* \\ Department of Mathematics, University of California, Berkeley, CA 94720, USA
}

1993

\begin{abstract}
This article is a follow-up of [3]. Its main goal is to provide an alternative proof of this part of the reconstruction theorem which concerns the existence of a connection. A construction of connection 1-form is presented. The formula expressing the local coefficients of connection in terms of the holonomy map is obtained as an immediate consequence of that construction. Thus the derived formula coincides with that used in $\llbracket$. The reconstruction and representation theorems form a generalization of the fact that the pointed configuration space of the classical Yang-Mills theory is equivalent to the set of all holonomy maps. The point of this generalization is that there is a one-to-one correspondence not only between the holonomy maps and the orbits in the space of connections, but also between all maps $\Omega M \rightarrow G$ fulfilling some axioms and all possible equivalence classes of $P(M, G)$ bundles with connection, where the equivalence relation is defined by bundle isomorphism in a natural way.
\end{abstract}

\section{Introduction}

Apart from purely mathematical motivation, which is to provide an alternative description for the moduli space of bundles with connections, there is also a physical incentive to study gauge theory by means of holonomy maps. The Aharonov-Bohm experiment indicates that neither a Yang-Mills potential (i.e. connection) nor a field strength (i.e. curvature) is an object corresponding directly to a given physical situation - connection possesses redundant degrees of freedom and curvature does not fully describe the situation in the case of a non-simply connected spacetime [6]. On the other hand, holonomy map can be measured and it provides more information than curvature [1, 2]. The reconstruction theorem gives the mathematical ground for treating holonomy as the primary, and potential and field strength (including the total space of the principal bundle on which they are defined) as the secondary, derived, objects of the theory [1, 3, 幽.

The holonomy, or loop space, approach was applied to derive the equations of motion of the nonabelian monopole [5, 7]. Furthermore, there is an extension of the holonomy formalism

*http://info.fuw.edu.pl/KMMF/ludzie_ang.htm (E-mail: pmh@fuw.edu.pl) 
used in Yang-Mills theory which is applied in general relativity. That extension, including physical motivations standing behind it, is described in [3]. With some negligible exceptions, the notations and conventions used throughout this paper are consistent with those of [3]. The next two paragraphs are devoted to establishing notation, recalling the reconstruction and representation theorems and the bundle construction. In paragraph 4, an alternative proof of the existence of the holonomy reconstructed connection is presented. An advantage of this proof is that it allows to write an explicit formula for the action of connection 1-form [see (5.1)] and, as a consequence, gives a geometrical interpretation for the local expression of the holonomy defined Yang-Mills potential [4.

\section{Notations and Conventions}

$\widetilde{\psi}$ family of paths: $\widetilde{\psi}: U \rightarrow P M, \widetilde{\psi}[u](i):=\psi(u, i)$, where $\psi: U \times[0,1] \rightarrow M$ is the associated map of $\widetilde{\psi} ; \widetilde{\psi}$ is called smooth iff $\psi$ is continuous and smooth on the subintervals $U \times\left[i_{n}, i_{n+1}\right]$ for $i_{0}=0<i_{1}<\ldots<i_{k}<i_{k+1}=1, n \in\{0, \ldots, k\}$

$a^{-1}$ diffeomorphism $: \pi^{-1}(\pi(a)) \rightarrow G$ defined by $a^{-1} b=g \Leftrightarrow b=a g$, where $\pi: P \rightarrow M$ is the projection of the principal fiber bundle $P(M, G)$

$\mathcal{F}$ the set of all triples $(P(M, G), \omega, b)$, where $M$ and $G$ are fixed and $\omega \in C(P), b \in \pi^{-1}(*)$

$\mathcal{F} / S$ generalized configuration space円; $S$ is an equivalence relation defined by:

$$
\begin{aligned}
& (P(M, G), \omega, b) S\left(P^{\prime}(M, G), \omega^{\prime}, b^{\prime}\right) \Longleftrightarrow \exists f \in \operatorname{Diff}\left(P, P^{\prime}\right): \pi^{\prime} \circ f=\pi, \\
& \forall g \in G: f \circ R_{g}=R_{g}^{\prime} \circ f, \quad\left(f^{-1}\right)^{*} \omega=\omega^{\prime}, \quad f(b)=b^{\prime}
\end{aligned}
$$

thin loop For precise definitions see p.1180 and p.1205 in [3], [8] and 6.1 in [9].

$\mathcal{H}$ the space of axiomatically defined holonomy maps:

$$
\mathcal{H}:=\{H: \Omega M \rightarrow G \mid H \text { satisfies axioms } 1,2,3\}
$$

Axiom $1 \forall \alpha, \beta \in \Omega M: H(\alpha \circ \beta)=H(\beta) H(\alpha)$ (The loop $\alpha \circ \beta$ is the one obtained by first going around the loop $\beta$ and then around $\alpha$.)

Axiom $2 H$ (any thin loop) $=e$ (Note that this axiom makes any two loops which differ only by their parametrization indistinguishable for any $H \in \mathcal{H}$.)

Axiom 3 For every smooth family of loops $\widetilde{\Omega}: U \rightarrow \Omega M, U$ an open subset of $\mathbb{R}^{n}, n \in \mathbb{N}$, $H \circ \widetilde{\Omega}: U \rightarrow G$ is smooth.

\footnotetext{
${ }^{1}$ If instead of fixing $M$ and $G$ we fix the entire bundle $P(M, G)$ and the point $b \in \pi^{-1}(*)$, then $\mathcal{F}$ will collapse to $C(P)$ and $\mathcal{F} / S$ will become the pointed configuration space of Yang Mills theory, i.e. $C(P) / G A_{*}(P)$, where $G A_{*}(P):=\left\{f \in \operatorname{Diff}(P) \mid \forall g \in G: f \circ R_{g}=R_{g} \circ f, \pi \circ f=\pi, f(b)=b\right\}$.
} 


\section{The Reconstruction and Representation Theorems and the Bundle Construction}

With notation assumed as above, the representation and reconstruction theorems are equivalent to:

Theorem 3.1 Let $M$ be a Hausdorff connected manifold and $H_{b}^{\omega}$ the holonomy map w.r.t. the point $b$ and connection $\omega$ (i.e. $\left.H_{b}^{\omega}: \Omega M \ni \alpha \mapsto \check{\alpha}(1)^{-1} \check{\alpha}(0), \pi \circ \check{\alpha}=\alpha, \check{\alpha}(0)=b, \omega \frac{d}{d i} \check{\alpha}(i)=0\right)$. The map

$$
h o l: \mathcal{F} / S \ni[(P(M, G), \omega, b)] \longmapsto H_{b}^{\omega} \in \mathcal{H}
$$

is well defined and bijective.

The representation part of Theorem 3.1 asserts that the map hol exists and is injective, whereas the reconstruction part claims that hol is surjective. The following are the geometric structures used to prove the theorem: $E:=(P M \times G) / T$ (total space), where $(p, g) T\left(p^{\prime}, g^{\prime}\right) \Longleftrightarrow(p(1)=$ $p^{\prime}(1)$ and $\left.g^{\prime}=H\left(p^{-1} \circ p^{\prime}\right) g\right),\{p, g\}=\left\{p^{\prime}, g^{\prime}\right\} \in E ; R_{g}\{p, h\}:=\{p, h g\}$ (right action of $G$ ); $\pi\{p, h\}:=p(1)$ (projection); $\widehat{p}(i):=\{K(p, i) \circ q, g\}$ (lifting of paths, to become the horizontal lifting), where $q \in P M, q(1)=p(0)$ and $K(p, i)$ is the contraction of $p$, i.e. $K(p, i)(j):=p(i j)$; local trivializations:

$$
\begin{gathered}
C_{\psi}: U \times G \stackrel{(\widetilde{\psi}, i d)}{\longrightarrow} P M \times G \longrightarrow \pi^{-1}(U) \subseteq(P M \times G) / T \\
C_{\psi}^{-1}\{p, g\}=C_{\psi}^{-1}\left\{\widetilde{\psi}[p(1)], H\left(p^{-1} \circ \widetilde{\psi}[p(1)]\right) g\right\}=\left(p(1), H\left(p^{-1} \circ \widetilde{\psi}[p(1)]\right) g\right),
\end{gathered}
$$

where $U$ is a contractible open subset of $M$ and $\widetilde{\psi}$ is a smooth family of paths $\widetilde{\psi}: U \rightarrow P M$ having the property $\forall u \in U: \widetilde{\psi}[u](1)=u$.

\section{Reconstruction of Connection}

Proposition 4.1 Let $\Gamma_{\{p, g\}}$ denote the vector subspace of $T_{\{p, g\}} E$ generated by vectors of the form $\left.\frac{d}{d i}\{K(r, i), h\}\right|_{i=j}$, where $h:=H\left(p^{-1} \circ K(r, j)\right) g$. Then the distribution $\Gamma: E \ni a \mapsto \Gamma_{a}$ is a smooth connection.

\section{Proof:}

a) $\forall\{p, g\} \in E: \Gamma_{\{p, g\}} \oplus \operatorname{Ker}\left(\pi_{*}: T_{\{p, g\}} E \rightarrow T_{p(1)} M\right)=T_{\{p, g\}} E$

That follows from the following lemmas:

\section{Lemma 1}

$$
\begin{aligned}
& \forall a \in E, X_{a} \in T_{a} E \exists p \in P M, k \in C^{\infty}([0,1], G): \\
& X_{a}=\left.\frac{d}{d i}\{K(p, i), k(j)\}\right|_{i=j}+\left.\frac{d}{d i}\{K(p, j), k(i)\}\right|_{i=j}
\end{aligned}
$$


Proof:

It is clear that for an arbitrary $X_{a}$ there exist smooth curves $\mathrm{p}$ and $\mathrm{g}$ such that $X_{a}=$ $\left.C_{\psi *} \frac{d}{d i}(p(i), g(i))\right|_{i=j}$. If we put $k(i)=H\left(\widetilde{\psi}[p(i)]^{-1} \circ K(p, i)\right) g(i)$, we have:

$$
\begin{aligned}
& X_{a}=\left.\frac{d}{d i}\{\widetilde{\psi}[p(i)], g(i)\}\right|_{i=j}=\left.\frac{d}{d i}\{K(p, i), k(i)\}\right|_{i=j}= \\
& =\left.C_{\psi *} \frac{d}{d i} C_{\psi}^{-1}\{K(p, i), k(i)\}\right|_{i=j}=\left.C_{\psi *} \frac{d}{d i}\left(p(i), H\left(K(p, i)^{-1} \circ \widetilde{\psi}[p(i)]\right) k(i)\right)\right|_{i=j}= \\
& =\left.C_{\psi *} \frac{d}{d i}\left(p(i), H\left(K(p, i)^{-1} \circ \widetilde{\psi}[p(i)]\right) k(j)\right)\right|_{i=j}+\left.C_{\psi *} \frac{d}{d i}\left(p(j), H\left(K(p, j)^{-1} \circ \widetilde{\psi}[p(j)]\right) k(i)\right)\right|_{i=j}= \\
& =\left.\frac{d}{d i}\left\{\widetilde{\psi}[p(i)], H\left(K(p, i)^{-1} \circ \widetilde{\psi}[p(i)]\right) k(j)\right\}\right|_{i=j}+\left.\frac{d}{d i}\left\{\widetilde{\psi}[p(j)], H\left(K(p, j)^{-1} \circ \widetilde{\psi}[p(j)]\right) k(i)\right\}\right|_{i=j}= \\
& =\left.\frac{d}{d i}\{K(p, i), k(j)\}\right|_{i=j}+\left.\frac{d}{d i}\{K(p, j), k(i)\}\right|_{i=j}
\end{aligned}
$$

Lemma $2 \forall a \in E: \pi_{*}: \Gamma_{a} \rightarrow T_{\pi(a)} M$ is an isomorphism.

Proof:

$$
\left.\pi_{*} \frac{d}{d i}\{K(p, i), h\}\right|_{i=j}=\left.\frac{d}{d i} p(i)\right|_{i=j} \Rightarrow \pi_{*} \text { is onto }
$$

Furthermore,

$$
\begin{gathered}
\left.\pi_{*} \frac{d}{d i}\{K(p, i), h\}\right|_{i=j}=\left.0 \Rightarrow \frac{d}{d i} p(i)\right|_{i=j}=0 \Rightarrow \\
\left.\frac{d}{d i}\{K(p, i), h\}\right|_{i=j}=\left.C_{\psi *} \frac{d}{d i}\left(p(i), H\left(K(p, i)^{-1} \circ \widetilde{\psi}[p(i)]\right) h\right)\right|_{i=j}= \\
=C_{\psi *}\left(0,\left.\frac{d}{d i}(H \circ \widetilde{\Omega})(p(i))\right|_{i=j}\right)=C_{\psi *}\left(0,\left.\partial_{\mu}(H \circ \widetilde{\Omega}) \frac{d}{d i} p^{\mu}(i)\right|_{i=j}\right)=0
\end{gathered}
$$

where $\widetilde{\Omega}[p(i)]:=K(p, i)^{-1} \circ \widetilde{\psi}[p(i)]$.

b) $R_{g *} \Gamma_{a}=\Gamma_{a g}($ trivial $)$

c) $\Gamma$ is smooth

Since smoothness is a local property, we are interested only in some open neighborhood of an arbitrarily chosen point $m \in M$ and therefore can treat that neighborhood as $\mathbb{R}^{n}, n:=\operatorname{dim} M, m=$ 0 . By Lemma 2 , it is enough to prove that $\forall \mu \in\{1, \ldots, n\}$ the lifting $\widehat{\partial}_{\mu}$ of the coordinate vector field $\partial_{\mu}$ is smooth. Let $\widetilde{\psi}$ and $\left\{\widetilde{\varphi_{\mu}}\right\}_{\mu \in\{1, \ldots, n\}}$ be smooth families of paths and $\left\{\widetilde{\Omega_{\mu}}\right\}_{\mu \in\{1, \ldots, n\}}$ smooth families of loops defined by:

$$
\psi(x, i)= \begin{cases}q(2 i) & \text { for } 0 \leq i \leq \frac{1}{2}, q \in P M, q(1)=0 \\ (2 i-1) x & \text { for } \frac{1}{2} \leq i \leq 1, x:=\left(x_{1}, \ldots, x_{n}\right) \in \mathbb{R}^{n}\end{cases}
$$




$$
\begin{aligned}
& \varphi_{\mu}(x, i)=\left\{\begin{array}{l}
q(4 i) \text { for } 0 \leq i \leq \frac{1}{4}, q \text { and } x \text { the same as above } \\
(4 i-1)\left(x_{1}, \ldots, x_{\mu}-\frac{1}{4}, \ldots, x_{n}\right) \text { for } \frac{1}{4} \leq i \leq \frac{1}{2} \\
\left(x_{1}, \ldots, x_{\mu}-\frac{3}{4}+i, \ldots, x_{n}\right)
\end{array}\right. \\
& \widetilde{\Omega_{\mu}}[(x, i)]=\widetilde{\psi}[x]^{-1} \circ T_{x, i}^{\mu} \circ \widetilde{\psi}\left[\widetilde{\varphi_{\mu}}[x](i)\right], \quad(x, i) \in \mathbb{R}^{n} \times\left(\frac{1}{2}, 1\right), \\
& T_{x, i}^{\mu}(j):=\widetilde{\varphi_{\mu}}[x](i)+j\left(x-\widetilde{\varphi_{\mu}}[x](i)\right)=(1-j)\left(x_{1}, \ldots, x_{\mu}-\frac{3}{4}+i, \ldots, x_{n}\right)+j x
\end{aligned}
$$

Clearly, $\widetilde{\psi}[x](1)=x$ and $\left.\frac{d}{d i} \widetilde{\varphi_{\mu}}[x](i)\right|_{i=\frac{3}{4}}=\partial_{\mu}(x)$. Since the differential structure on $E$ is given by its local trivializations and $C_{\psi}$ is a local trivialization of $E$, it suffices to show that $C_{\psi *}^{-1} \widehat{\partial_{\mu}}$ is a smooth vector field on $\mathbb{R}^{n} \times G$ :

$$
\begin{aligned}
& \left(C_{\psi *}^{-1} \widehat{\partial_{\mu}}\right)(x, g)=C_{\psi *}^{-1} \widehat{\partial_{\mu}}\left(C_{\psi}(x, g)\right)=C_{\psi *}^{-1} \widehat{\partial_{\mu}}(\{\widetilde{\psi}[x], g\})= \\
& =\left.C_{\psi *}^{-1} \frac{d}{d i}\left\{K\left(\widetilde{\varphi_{\mu}}[x], i\right), H\left(\widetilde{\psi}[x]^{-1} \circ K\left(\widetilde{\varphi_{\mu}}[x], \frac{3}{4}\right)\right) g\right\}\right|_{i=\frac{3}{4}}= \\
& =\left.\frac{d}{d i}\left(\widetilde{\varphi_{\mu}}[x](i), H\left(K\left(\widetilde{\varphi_{\mu}}[x], i\right)^{-1} \circ \widetilde{\psi}\left[\widetilde{\varphi_{\mu}}[x](i)\right]\right) H\left(\widetilde{\psi}[x]^{-1} \circ K\left(\widetilde{\varphi_{\mu}}[x], \frac{3}{4}\right)\right) g\right)\right|_{i=\frac{3}{4}}= \\
& =\left(\partial_{\mu}(x),\left.\frac{\partial}{\partial i}\left(H \circ \widetilde{\Omega_{\mu}}\right)(x, i) g\right|_{i=\frac{3}{4}}\right)
\end{aligned}
$$

Now, the smoothness of $C_{\psi *}^{-1} \widehat{\partial_{\mu}}$ follows from the smoothness of $H \circ \widetilde{\Omega_{\mu}}$.

\section{Connection 1-form}

Let $P_{V}$ denote the projection on vertical subspaces and $X_{a}=\left.\frac{d}{d i}\{\tilde{\chi}[i], g(i)\}\right|_{i=j}$ be an arbitrary vector tangent to $E$. The action of connection 1-form on $X_{a}$ can be described as follows:

$$
\omega_{a}: T_{a} E \ni X_{a} \longmapsto\left(a^{-1}\right)_{*} P_{V} X_{a} \in G^{\prime}
$$

By Lemma 1, we have

$$
X_{a}=\left.\frac{d}{d i}\{K(p, i), k(j)\}\right|_{i=j}+\left.\frac{d}{d i}\{K(p, j), k(i)\}\right|_{i=j},
$$

where $p(i):=\tilde{\chi}[i](1), k(i):=H\left(\widetilde{\chi}[i]^{-1} \circ K(p, i)\right) g(i)$.

Hence

$$
\begin{aligned}
& \omega_{a} X_{a}=\left.\left(\{K(p, j), k(j)\}^{-1}\right)_{*} \frac{d}{d i}\{K(p, j), k(i)\}\right|_{i=j}= \\
& =\left.\frac{d}{d i}\{K(p, j), k(j)\}^{-1}\{K(p, j), k(i)\}\right|_{i=j}=\left.\frac{d}{d i} k(j)^{-1} k(i)\right|_{i=j}= \\
& =\left.\frac{d}{d i}\left(H\left(\tilde{\chi}[j]^{-1} \circ K(p, j)\right) g(j)\right)^{-1} H\left(\tilde{\chi}[i]^{-1} \circ K(p, i)\right) g(i)\right|_{i=j}=
\end{aligned}
$$




$$
\begin{aligned}
& =\left.g(j)^{-1} \frac{d}{d i} H\left(K(p, j)^{-1} \circ \tilde{\chi}[j]\right) H\left(\tilde{\chi}[i]^{-1} \circ K(p, i)\right) g(i)\right|_{i=j}= \\
& =\left.g(j)^{-1} \frac{d}{d i} H\left(\tilde{\chi}[i]^{-1} \circ K(p, i) \circ K(p, j)^{-1} \circ \tilde{\chi}[j]\right) g(i)\right|_{i=j}
\end{aligned}
$$

Note that $K(p, i) \circ K(p, j)^{-1}$ is a curve beginning at $p(j)$ and ending at $p(i)$ whose image is contained in the image of $p$.

To compute the local coefficients of $\omega$ we must pick up a local section of $E$. The natural choice seems to be:

$$
\sigma: U \ni x \longmapsto C_{\psi}(x, e) \in \pi^{-1}(U),
$$

where $C_{\psi}$ is a local trivialization over $U$. Similarly as in the proof of the smoothness of $\Gamma$, we are interested only in some open subset of $M$ which is small enough to be diffeomorphic to $\mathbb{R}^{n}$. Therefore, in what follows, $U$ will be identified with $\mathbb{R}^{n}$. Putting $T_{y, x}(i)=x+i(y-x)$ and $T_{y_{\mu}, x}(i)=x+i\left(0, \ldots, y_{\mu}-x_{\mu}, \ldots, 0\right)$ and using (5.1) we get:

$$
\begin{aligned}
& A_{\mu}(x):=\left(\sigma^{*} \omega\right)_{x} \partial_{\mu}(x)=\omega_{\sigma(x)} \sigma_{*} \partial_{\mu}(x)=\left.\omega_{\sigma(x)} \sigma_{*} \frac{d}{d y_{\mu}} T_{y_{\mu}, x}(1)\right|_{y_{\mu}=x_{\mu}}= \\
& =\left.\omega_{\sigma(x)} \frac{d}{d y_{\mu}}\left\{\widetilde{\psi}\left[T_{y_{\mu}, x}(1)\right], e\right\}\right|_{y_{\mu}=x_{\mu}}=\left.\frac{d}{d y_{\mu}} H\left(\widetilde{\psi}\left[T_{y_{\mu}, x}(1)\right]^{-1} \circ T_{y_{\mu}, x} \circ \widetilde{\psi}\left[T_{x_{\mu}, x}(1)\right]\right)\right|_{y_{\mu}=x_{\mu}}= \\
& =\left.\frac{\partial}{\partial y_{\mu}} H\left(\widetilde{\psi}[y]^{-1} \circ T_{y, x} \circ \widetilde{\psi}[x]\right)\right|_{y=x}
\end{aligned}
$$

Formula (5.2) coincides with formula (5.26) in [四], where it is taken to be the definition of the gauge potential $A_{\mu}$.

\section{Example}

Let $M=\mathbb{R}^{2}, *=0, G=\mathbb{R}_{*}$ and $H(\beta)=\exp \int_{\beta} y d x, \beta \in \Omega \mathbb{R}^{2}$. Clearly, the reconstructed bundle $E$ is trivial and can be identified with $\mathbb{R}^{2} \times \mathbb{R}_{*}$ via the bundle isomorphism $C_{\psi}: \mathbb{R}^{2} \times \mathbb{R}_{*} \rightarrow$ $E$, where $\psi(x, y, i):=i(x, y)$. Due to the invariance of connection 1-forms under the right action of $\mathbb{R}_{*}$, the reconstructed connection 1-form pulled back to $\mathbb{R}^{2} \times \mathbb{R}_{*}$ can be written as:

$$
\left(C_{\psi}^{*} \omega\right)_{(x, y, z)}=A_{1}(x, y) d x+A_{2}(x, y) d y+\frac{A_{3}(x, y)}{z} d z
$$

Furthermore,

$$
\begin{aligned}
& \quad \frac{A_{3}(x, y)}{z}=C_{\psi}^{*} \omega \frac{\partial}{\partial z}(x, y, z)=\left.C_{\psi}^{*} \omega \frac{d}{d i}(x, y, z+i)\right|_{i=0}= \\
& =\left.\omega \frac{d}{d i} C_{\psi}(x, y, z+i)\right|_{i=0}=\left.\omega \frac{d}{d i}\{\widetilde{\psi}[(x, y)], z+i\}\right|_{i=0}= \\
& =\left.\left(\{\widetilde{\psi}[(x, y)], z\}^{-1}\right)_{*} \frac{d}{d i}\{\widetilde{\psi}[(x, y)], z+i\}\right|_{i=0}=\left.\frac{d}{d i} \frac{z+i}{z}\right|_{i=0}=\frac{1}{z},
\end{aligned}
$$


i.e. $A_{3}(x, y) \equiv 1$. Now, let $\sigma: \mathbb{R}^{2} \rightarrow \mathbb{R}^{2} \times \mathbb{R}_{*}$ be a global section defined by $\sigma(x, y)=(x, y, 1)$. Using (6.1) and then (5.2), we obtain:

$$
\begin{aligned}
& A_{1}\left(x_{0}, y_{0}\right)=\sigma^{*} C_{\psi}^{*} \omega \frac{\partial}{\partial x}\left(x_{0}, y_{0}\right)=\left(C_{\psi} \circ \sigma\right)^{*} \omega \frac{\partial}{\partial x}\left(x_{0}, y_{0}\right)= \\
& =\left.\frac{d}{d x} H\left(\widetilde{\psi}\left[\left(x, y_{0}\right)\right]^{-1} \circ T_{x,\left(x_{0}, y_{0}\right)} \circ \widetilde{\psi}\left[\left(x_{0}, y_{0}\right)\right]\right)\right|_{x=x_{0}}=\left.\frac{d}{d x} \exp \frac{y_{0}}{2}\left(x-x_{0}\right)\right|_{x=x_{0}}=\frac{y_{0}}{2}
\end{aligned}
$$

Similarly,

$$
A_{2}\left(x_{0}, y_{0}\right)=\left.\frac{d}{d y} H\left(\widetilde{\psi}\left[\left(x_{0}, y\right)\right]^{-1} \circ T_{y,\left(x_{0}, y_{0}\right)} \circ \widetilde{\psi}\left[\left(x_{0}, y_{0}\right)\right]\right)\right|_{y=y_{0}}=\left.\frac{d}{d y} \exp \frac{-x_{0}}{2}\left(y-y_{0}\right)\right|_{y=y_{0}}=\frac{-x_{0}}{2}
$$

Hence $\left(C_{\psi}^{*} \omega\right)_{(x, y, z)}=\frac{1}{2} y d x-\frac{1}{2} x d y+z^{-1} d z$.

Acknowledgments: I am indebted to Andrzej Borowiec and Joseph Wolf for discussions concerning this article as well as for their encouragement. I also would like to thank Arkadiusz Jadczyk for drawing my attention to the subject.

\section{References}

[1] Anandan, J., "Holonomy Groups in Gravity and Gauge Fields", in Conference on Differential Geometric Methods in Theoretical Physics, G. Denardo and H. D. Doebner, eds., World Scientific, Singapore, 1983.

[2] Anandan, J., "Gauge Fields, Quantum Interference and Holonomy Transformations." Physical Review D, vol.33, No.8, 2262-2266 (1986).

[3] Barrett, J. W., "Holonomy and Path Structures in General Relativity and Yang-Mills Theory." International Journal of Theoretical Physics, vol.30, No.9 (1991).

[4] Chan, H.-M., Scharbach, P. and Tsou S.T., "On Loop Space Formulation of Gauge Theories." Annals of Physics, 166, 396-421 (1986).

[5] Chan, H.-M., Scharbach, P. and Tsou S.T., "Action Principle and Equations of Motion for Nonabelian Monopoles." Annals of Physics, 167, 454-472 (1986).

[6] Chan, H.-M., and Tsou S.T., "Gauge Theories in Loop Space." Acta Physica Polonica B, 17, 259-276 (1986).

[7] Chan, H.-M., and Tsou S.T., Dual Yang-Mills Theory - the Quantum Theory of Nonabelian Monopoles, Rutherford Appleton Preprint RAL-92-036.

[8] Kobayashi, S., "La connexion des variétés fibrées." Comptes Rendus, 238, 443-444 (1954).

[9] Menskii, M. B., Gruppa Putei, Izmereniia, Polia, Chastitsy, Nauka, Moskva, 1983.

For more references and a discussion concerning the development of the subject see [3]. 